Fanggeng Zou $\cdot$ Yucheng Peng $\cdot$ Xiaoyan Wang

Aihua Sun - Wanqing Liu • Shaochun Bai $\cdot$ Hui Zhu

Bo Gao - Guoyin Feng $\cdot$ Lin He

\title{
A locus for congenital preauricular fistula maps to chromosome 8q11.1-q13.3
}

Received: October 16, 2002; Accepted: November 25, 2002

\begin{abstract}
The incidence of congenital preauricular fistula (CPF) is $>1.1 \%$ in both Chinese and Caucasians, but it is even higher in Blacks. We mapped the locus for CPF to chromosome 8q11.1-q13.3 by linkage analysis of a family composed of 7 affected and 11 nonaffected members. The two-point LOD score was 2.40 , shown by markers D8S285 and D8S1113 at a recombination fraction $(\theta)$ of 0.00 . Results from three other markers (D8S1110, D8S260, and D8S1136) in the same region further support the linkage. Haplotype analysis for this family confined the locus to within an interval of approximately $26.7 \mathrm{cM}$, flanked by markers D8S532 and D8S279. A LOD score of $<3$ is likely due to the limitation of family size.
\end{abstract}

Key words Congenital preauricular fistula $\cdot$ Linkage analysis $\cdot$ Gene mapping $\cdot$ Genotyping $\cdot$ Haplotyping $\cdot$ Mutation

\section{Introduction}

Congenital preauricular fistula (CPF, MIM 128700) is a common auricular abnormality, the incidence of which is $1.2 \%$ in Chinese (He and Jiang 1983), 1.1\% in Caucasians

F. Zou $\cdot$ X. Wang $\cdot$ W. Liu $\cdot$ S. Bai $\cdot$ H. Zhu $\cdot$ L. He

Shanghai Research Center of Life Sciences, Chinese Academy of Sciences, Shanghai, China

H. Zhu $\cdot$ B. Gao $\cdot$ G. Feng $\cdot$ L. He $(\bowtie)$

Bio-X Life Science Research Center, P.O. Box 501, Hao Ran Building, Shanghai Jiao Tong University, 1954 Hua Shan Road, Shanghai 200030, P.R. China

Tel. +86-21-62822491; Fax +86-21-62822491

e-mail: helin@sjtu.edu.com

Y. Peng $\cdot$ A. Sun

Shanghai Chang Zheng Hospital, Shanghai, China

The first and third authors contributed equally to this work.
(Gualandri 1969), and higher in Blacks (Simpkiss and Lowe 1961). Familial CPF associated with major congenital disorders is not observed, whereas serious congenital anomalies have been detected in one third of sporadic cases of CPF (Meggyessy and Mehes 1982). CPF occurs in the upper anterior end of the ear helix as a kind of narrow, winding fistula, sometimes with branches. The formation of CPF is closely associated with embryological development of the auricle, which is developed from the first and second branchial arches. It is hypothesized that this is either due to malfusion of the cumular nodules of the first and second branchial arches, or due to incomplete closure of the first branchial cleft (He and Jiang 1983). CPF is generally classified into the following three types: simple, infectious, and secretory. The majority of patients are seen by physicians because of infection, but the simple and secretory types of $\mathrm{CPF}$ are usually also found in patients with infectious CPF. Gualandri (1969) found 321 cases of CPF in 29309 school children in Milan, 93 of whom had a condition of autosomal dominant inheritance with high penetrance. Another report based on a large kindred also suggested this mode of inheritance (Bhalla et al. 1979). However, the gene underlying CPF has not been assigned.

Here we report the result of a genome-wide linkage analysis on a Chinese family with CPF.

\section{Patients and methods}

Family and patients

A Chinese family with three generations comprising 18 members ( 7 affected and 11 unaffected individuals) from Shanghai was recruited in this study. Informed consent was obtained from all the family members for participation in a linkage analysis, and they underwent general medical and detailed otorhinolaryngologic evaluations in Shanghai Chang Zheng Hospital. Except for preauricular fistulae, they did not express congenital defects such as hearing impairment, renal dysplasia, and branchial fistulae, which 
are all characteristic of branchio-oto-renal (BOR) syndrome. Results of clinical evaluations showed that the affected family members had simple preauricular fistulae but not any syndromes. Among the seven affected members, individuals II-1 and II-8 (Fig. 1) showed a preauricular fistula on the left ear, whereas individuals II-6, II-11, III-1, III-6, and III-7 had the fistulae bilaterally.

\section{Genotyping and linkage analysis}

Peripheral blood samples were taken from all available members in the family, and DNA was extracted by the standard method (Yang et al. 2000). A set of dinucleotide repeat microsatellite markers based on the Généthon genetic map (Dib et al. 1996), with a 10-cM resolution, was purchased from Perkin-Elmer Applied Biosystems (Foster City, CA, USA) and recruited for genome-wide genotyping. We also selected additional markers for denser mapping of candidate chromosomal regions. The forward primer of each primer pair was labeled with fluorescent dyes (FAM, HEX, or NED) to perform multiplex analysis on an ABI 377 DNA sequencer (Perkin-Elmer Applied Biosystems).

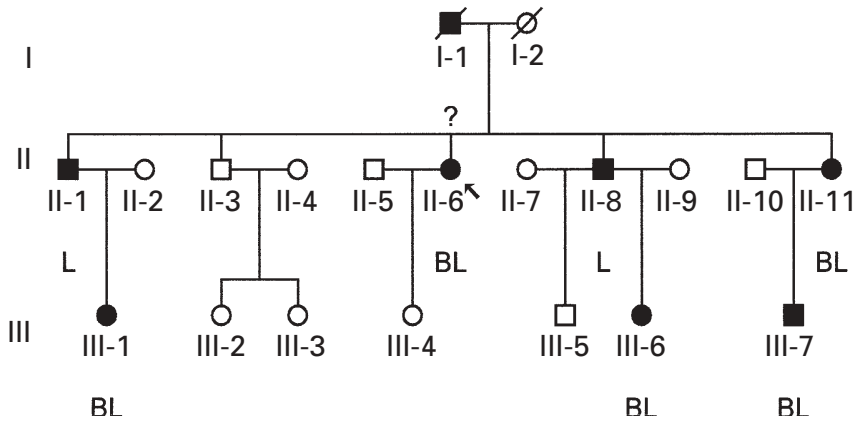

Fig. 1. Pedigree of congenital preauricular fistula. Open, solid, square, and circle symbols are unaffected, affected, men, and women, respectively. $B L$, Bilaterally affected; $L$, affected in the left ear only. The affected side of individual I-1 was unknown
Two-point LOD scores for the putative disease locus were calculated in each marker by the use of the MLINK program of FASTLINK version 5.1 (Lathrop et al. 1984). An autosomal dominant model with $99 \%$ penetrance was assumed, and the disease-gene frequency was set at 0.001 for the LOD score calculation. LOD scores were calculated at recombination fractions $(\theta)$ of $0.00,0.01,0.05,0.10,0.20$, 0.30 , and 0.40 . Equal recombination frequencies were assumed between male and female family members. The maximum score $(\mathrm{Zmax})$ was calculated by the ILINK program of the LINKAGE software package.

In a candidate gene, $E Y A 1$, we sequenced all 17 exons using an ABI 3100 DNA sequencer (Perkin-Elmer Applied Biosystems; Gao et al. 2001). Primers were synthesized according to the sequences published (Abdelhak et al. 1997; Vincent et al. 1997; Kumar et al. 1998; Azuma et al. 2000).

\section{Results}

By carrying out genome-wide scanning, two adjacent markers (D8S285 and D8S260) at chromosome 8q gave LOD scores of 2.40 and 2.10, respectively. Further analysis of four markers (D8S1110, D8S1113, D8S1136, and D8S2324) also strongly supported this region as a candidate. The highest LOD score $(Z \max )$ of 2.40 at $\theta=0.00$ was obtained at marker loci D8S285 and D8S1113. D8S260 and D8S1136 adjacent to D8S1113 also revealed a LOD score of $>2$. More markers (D8S505, D8S1722, D8S532, D8S543, D8S279, and D8S275) recruited to define the CPF locus in the denser map resulted in proportional positive scores according to their locations (Table 1).

Haplotypes of the family were constructed with all the markers listed earlier (Fig. 2). A disease-associated haplotype shared by all the affected individuals (II-1, II-6, II-8, II-11, III-1, III-6, and III-7) was observed. Recombination events were found in two affected individuals (III-6 and III-7) and in two unaffected individuals (III-4 and III-5). Based on these recombination events, we confined the CPF

Table 1. Two-point linkage data in the CPF family

\begin{tabular}{|c|c|c|c|c|c|c|c|c|c|}
\hline \multirow[b]{2}{*}{ Locus } & \multicolumn{7}{|c|}{ LOD score at recombination fraction $(\theta)$} & \multirow[b]{2}{*}{ Zmax } & \multirow[b]{2}{*}{$\theta \max$} \\
\hline & .00 & .01 & .05 & .10 & .20 & .30 & .40 & & \\
\hline D8S505 & -3.19 & -0.23 & 0.34 & 0.48 & 0.43 & 0.25 & 0.07 & 0.49 & .126 \\
\hline D8S1722 & -2.59 & 0.36 & 0.90 & 0.99 & 0.83 & 0.50 & 0.14 & 0.99 & .10 \\
\hline D8S532 & -2.54 & 0.36 & 0.90 & 0.99 & 0.83 & 0.51 & 0.15 & 0.99 & .10 \\
\hline D8S1110 & 1.20 & 1.18 & 1.07 & 0.93 & 0.63 & 0.33 & 0.08 & 1.20 & .00 \\
\hline D8S285 & 2.40 & 2.35 & 2.18 & 1.94 & 1.43 & 0.86 & 0.28 & 2.40 & .00 \\
\hline D8S1113 & 2.40 & 2.35 & 2.18 & 1.94 & 1.43 & 0.86 & 0.28 & 2.40 & .00 \\
\hline D8S260 & 2.10 & 2.06 & 1.90 & 1.69 & 1.23 & 0.72 & 0.22 & 2.10 & .00 \\
\hline D8S1136 & 2.10 & 2.06 & 1.90 & 1.69 & 1.23 & 0.72 & 0.22 & 2.10 & .00 \\
\hline D8S543 & 0.92 & 0.90 & 0.82 & 0.72 & 0.50 & 0.28 & 0.09 & 0.92 & .00 \\
\hline D8S279 & 0.29 & 0.56 & 0.88 & 0.93 & 0.76 & 0.45 & 0.12 & 0.94 & .092 \\
\hline D8S2324 & -0.99 & -0.67 & -0.17 & 0.06 & 0.18 & 0.13 & 0.04 & 0.18 & .204 \\
\hline D8S275 & -4.42 & -2.38 & -1.33 & -0.81 & -0.31 & -0.11 & -0.02 & -0.00 & .501 \\
\hline
\end{tabular}

$\mathrm{CPF}$, Congenital preauricular fistula 
Fig. 2. The pedigree of congenital preauricular fistula showing haplotypes for polymorphic markers at 8q11.1-13.3. Marker orders were determined from the généthon sex-averaged genetic map and Genome Database. Open, solid, square, and circle symbols are unaffected, affected, men, and women, respectively. The haplotype shared by affected members is boxed

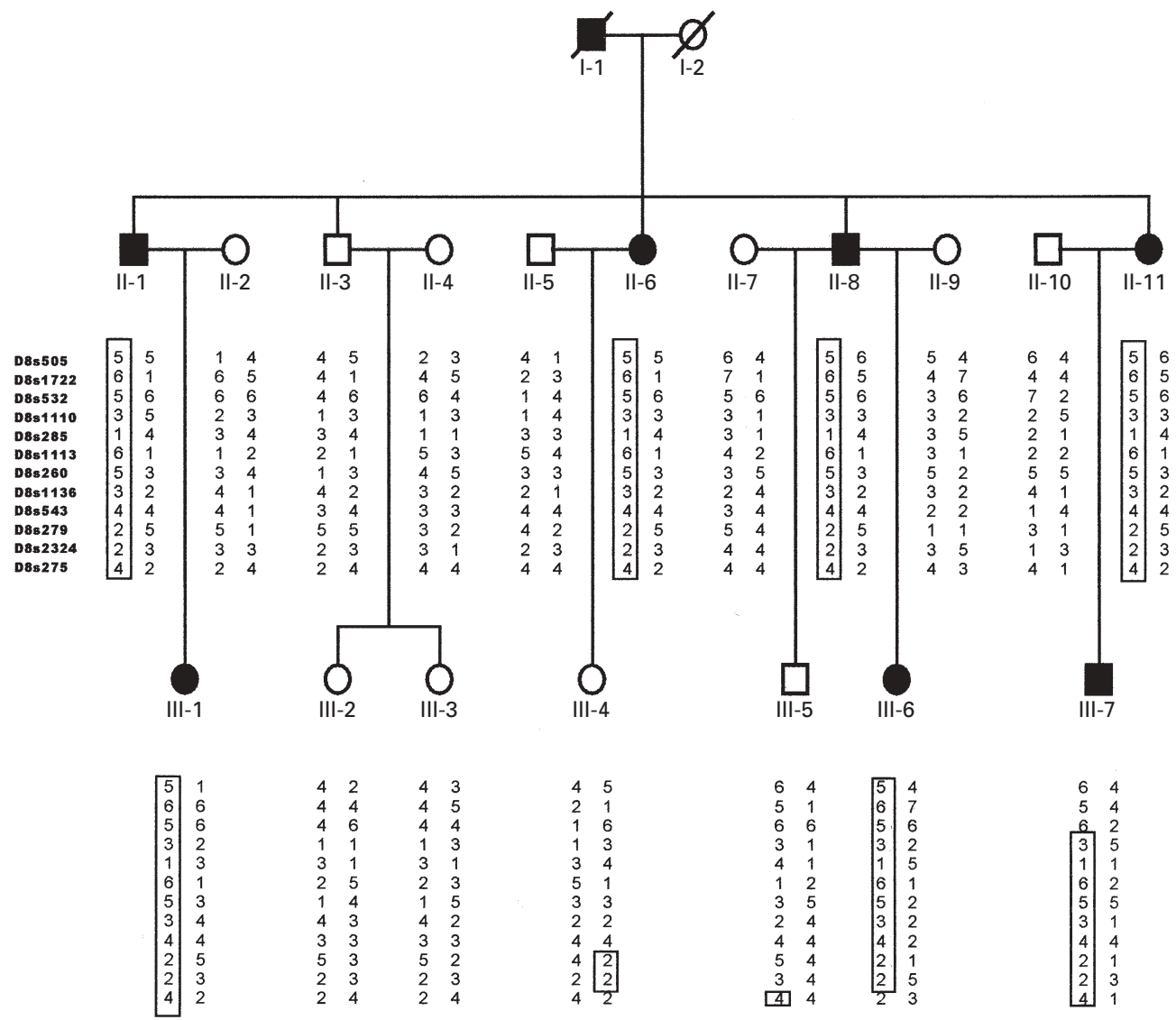

locus to an interval of $<26.7 \mathrm{cM}$ flanked by markers D8S532 and D8S279 at 8q11.1-q13.3.

No mutations in EYAl were found in any affected members in the family.

\section{Discussion}

We successfully assigned the CPF locus to 8q11.1-q13.3. Because of the limitation of the size of the family we analyzed, the Zmax obtained was 2.40, lower than a significant value of 3.00. Positive results at multiple markers within the region, as well as the shared haplotype by all the affected members, strongly supported the linkage between the disease locus and the marker loci. In addition, the critical recombinants narrowed the linked interval to approximately $26.7 \mathrm{cM}$ near markers D8S532 and D8S279.

The putative CPF gene may have functions in the developmental process of the auricle. By searching the database (Dib et al. 1996) for candidate genes in the region between D8S532 and D8S279, we considered EYA1, the human homologue of the Drosophila eyes absent gene, as the most likely candidate gene for CPF. EYA1 has been reported to be responsible for BOR syndrome or branchial-otic syndrome (Abdelhak et al. 1997; Vincent et al. 1997). BOR syndrome, first described by Melnick et al. (1976), is characterized by branchial anomalies affecting the outer middle and/or inner ear that frequently lead to sensorineural, con-

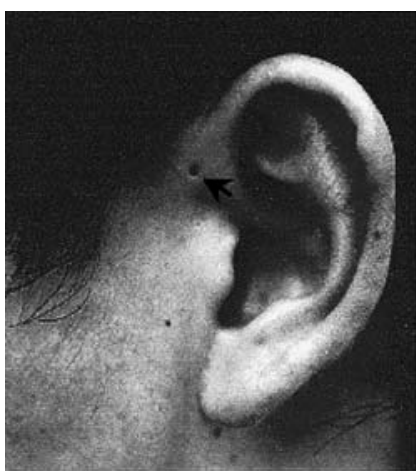

A

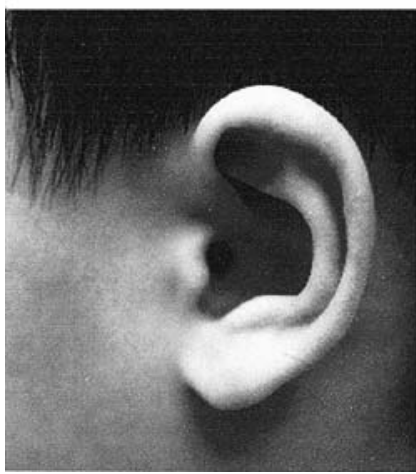

C

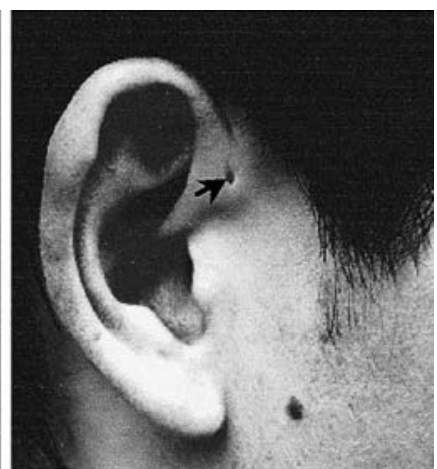

B

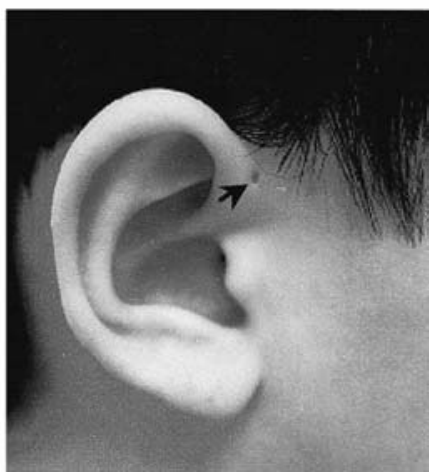

D
Fig. 3.A-D. Congenital preauricular fistula in a bilaterally affected member (A and $\mathbf{B})$ and in a unilaterally affected member $(\mathbf{C}$ and $\mathbf{D})$ 
ductive, or mixed hearing loss (Fraser et al. 1980), and by a wide spectrum of renal anomalies ranging form mild hypoplasia to lethal bilateral renal aplasia. We found no mutation in EYA1 in affected members in our family. However, because BOR syndrome and CPF are both heterogeneous, we cannot fully exclude $E Y A 1$ from the candidacy for CPF in our family.

Interestingly, we have found phenotypes to be discordant between generations in this family (Figs. 1 and 3). For example, the fistula of II-1 appeared on his left ear, whereas those of his daughter (III-1) were bilateral. Bilateral fistulae appeared in both II-11 and his son (III-7). A similar phenomenon was also found in some other family members. We performed statistical analysis in $54 \mathrm{CPF}$ families (no DNA samples available), and clinical information was collected by a physician at Chang Zheng Hospital in Shanghai. Of 186 affected individuals, 91 had CPF bilaterally and 95 had CPF unilaterally (left and right ear involvement in 48 and in 47 individuals, respectively). Because we were not able to find any distinct patterns in the study, a larger number of families may be required to further our understanding of CPF.

Acknowledgments We would like to thank Shanghai Municipal Commission for Science and Technology, the National Natural Science Foundation of China, and the National 973 and 863 Projects for their generous financial support of this study.

\section{References}

Abdelhak S, Kalatzis V, Heilig R, Compain S, Samson D, Vincent C (1997) A human homologue of the Drosophila eyes absent gene underlies branchio-oto-renal (BOR) syndrome and identifies a novel gene family. Nat Genet 15:157-164
Azuma N, Hirakiyama A, Inoue T, Asaka A, Yamada M (2000) Mutations of a human homologue of the Drosophila eyes absent gene (EYA1) detected in patients with congenital cataracts and ocular anterior segment anomalies. Hum Mol Genet 9:363-366

Bhalla V, Roy S, Inam AS (1979) Familial transmission of preauricular fistula in a seven generation Indian pedigree. Hum Genet 48: 339-341

Cannon FE (1941) Inheritance of ear pits in six generations of a family. J Hered 32:413-414

Dib C, Faure S, Fizames C, Samson D, Drouot N, Vignal A, Millasseau $\mathrm{P}$ (1996) A comprehensive genetic map of the human genome based on 5264 microsatellites. Nature 380:152-154

Fraser FC, Sproule JR, Halal F (1980) Frequency of the branchiooto-renal (BOR) syndrome in children with profound hearing loss. Am J Med Genet 7:341-349

Gao B, Guo J, She C, Shu A, Yang M, Tan Z, Yang X, Guo S, Feng G, $\mathrm{He}$ L (2001) Mutations in IHH, encoding Indian hedgehog, cause brachydactyly type A-1. Nat Genet 28:386-388

Gualandri V (1969) Richerche genetiche sulla fistula auris congenita. Acta Genet Med Gemellol (Roma) 18:51-68

He YZ, Jiang SC (1983) Otology. Shanghai Science and Technology Shanghai, pp 379-382

Kumar S, Kimberling WJ, Weston MD, Schaefer BG, Berg MA (1998) Identification of three novel mutations in human EYA1 protein associated with branchio-oto-renal syndrome. Hum Mutat 11: 443-449

Lathrop GM, Lalouel JM, Julier C, Ott J (1984) Easy calculation of LOD scores and genetic risks on small computers. Am J Hum Genet 36:460-465

Meggyessy V, Mehes K (1982) Preauricular pits in Hungary: epidemiologic and clinical observations. J Craniofac Genet Dev Biol 2: 215-218

Melnick M, Bixler D, Nance W, Silk K, Yune H (1976) Familial branchial-oto-renal dysplasia: a new addition to the branchial arch syndromes. Clin Genet 9:25-34

Simpkiss MJ, Lowe A (1961) Congenital abnormalities in the African newborn. Arch Dis Child 36:404-406

Vincent C, Kalatzis V, Abdelhak S, Chaib H, Compain S (1997) BOR and BO syndrome are allelic defects of EYA1. Eur J Hum Genet 5:242-246

Yang X, She C, Guo J, Yu A CH, Lu Y, Shi X, Feng G, He L (2000) A locus for brachydactyly type A-1 maps to chromosome 2q35-q36. Am J Hum Genet 66:892-903 